\title{
Wavelet analysis of blood flow dynamics: effect on the individual oscillatory components of iontophoresis with neutral electrolytes
}

\author{
Mitja Veber $\dagger$ Andriy Bandrivskyy $\ddagger$, Peter B M Clarkson§, \\ Peter V E McClintock $\$ \|$ and Aneta Stefanovska $\dagger$ \\ $\dagger$ Group of Nonlinear Dynamics and Synergetics, Faculty of Electrical \\ Engineering, University of Ljubljana, Tržaška 25, 1000 Ljubljana, Slovenia \\ ¥ Department of Physics, Lancaster University, Lancaster, LA1 4YB, UK \\ $\S$ Cardiology Department, Royal Lancaster Infirmary, Ashton Road, Lancaster \\ LA1 4RP, UK
}

\begin{abstract}
Iontophoresis currents are used in the transcutaneous delivery of vasoactive substances for noninvasive assessment of skin vascular properties. The blood flow rate can be recorded by laser Doppler flowmetry (LDF), its average value and the amplitudes of its oscillatory components being used to evaluate the effect of the drugs. Because non-drug-specific, current-induced, vasodilation could confound the interpretation of the response, we have investigated the effect of currents of both polarities on the spectral components of the LDF signal in the absence of vasoactive substances. It was recorded for healthy volunteers with both high conductance $(5 \mathrm{~mol} / \mathrm{l} \mathrm{NaCl})$ and low conductance (deionised water) electrolytes. The oscillatory components were analysed by wavelet transform within $0.0095-1.6 \mathrm{~Hz}$, divided into 5 sub-intervals. Only cathodal iontophoresis with deionised water increased the oscillatory energy and amplitude. It did so at all frequencies, but none of the sub-intervals associated with vasodilation $(0.0095-$ $0.145 \mathrm{~Hz}$ ) was selectively affected compared to the others.
\end{abstract}

Submitted to: Phys. Med. Biol.

\section{Introduction}

The coupled oscillator model (?) of the cardiovascular system has the advantage that individual oscillatory components of physiological signals can plausibly be associated with particular physiological processes that express dominantly in specific frequency intervals: components at $\sim 1 \mathrm{~Hz}$ and $\sim 0.2 \mathrm{~Hz}$ correspond to heart and respiration; that at $\sim 0.1 \mathrm{~Hz}$ probably derives from intrinsic myogenic activity of smooth muscle (?); and there is compelling evidence that those at $\sim 0.03$ and $\sim 0.01 \mathrm{~Hz}$ correspond respectively to sympathetic neurogenic activity (SNA) (?) and endothelial-related (see below) processes. Due to mutual coupling the effect of one physiological process may manifest in several frequency intervals, or several physiological processes may be involved in maintaining a basic oscillatory activity expressed within one frequency interval. For example, new experimental approaches are revealing a possible role for 
arterial baroreceptors in the chronic regulation of SNA (?). Spectral analysis can be used to separate the components in e.g. blood flow signals. Once the physiological origin of the underlying oscillatory processes has been fully understood, studies of the amplitudes and couplings provide in principle a noninvasive means of obtaining detailed information about the physiological state of the cardiovascular system.

Vasoactive substances can be used to assess vascular properties through their selective effect on the different physiological processes. Such drugs can be introduced locally, using iontophoresis to transport them across the dermal barrier as unipolar currents of relatively large ionized molecules (?????). Laser Doppler flowmetry (LDF) can then be used to study the local blood flow responses (??). Vasodilation induced by iontophoretically administered acetylcholine $(\mathrm{ACh})$ results in a higher relative magnitude of the oscillatory component near $0.01 \mathrm{~Hz}$ than that induced by sodium nitroprusside (SNP) (???). Given that $\mathrm{ACh}$ is an endothelium-dependent vasodilator, whereas SNP (an NO donor) influences vascular activity directly independently of the endothelium, these results can be interpreted as evidence that the $0.01 \mathrm{~Hz}$ oscillatory component is related to endothelial activity. Through comparison of the responses to ACh and SNP, iontophoresis may be used to study the dysfunction of vascular endothelium in atherosclerosis, essential hypertension, diabetes mellitus, heart failure and other vascular diseases.

Unfortunately, analyses of this kind have been subject to a continuing uncertainty of interpretation in view of long-standing evidence of significant vasodilation with neutral electrolytes such as water (?????). From analyses of average LDF signals (??), it is known that the current polarity affects vasodilation for low conductance electrolytes (deionised water), with cathode current producing a larger effect than anode current. It is obviously necessary to be able to distinguish the non-drug-specific effect of the current itself on the oscillatory components in the LDF signal.

The aim of this Note is to report an investigation of current-induced vasodilation with neutral electrolytes in which we seek to establish whether or not individual oscillatory components are selectively affected by the current. It is, in effect, the essential control experiment needed to confirm the conclusions drawn in earlier studies e.g. of the vascular endothelium (???) and to underpin future investigations being planned in several laboratories.

\section{Methods}

Fourteen healthy males aged $22-60$ years $(29.4 \pm 10.7$ years) gave their informed written consent to the study, which was performed according to the Declaration of Helsinki under procedures approved by the Morecambe Bay Local Ethics Committee. None of them was taking vasoactive medication, and they were asked neither to smoke nor to consume caffeine-containing drinks on the days of measurements.

The same iontophoretic apparatus, procedure and current density $\left(2 \mu \mathrm{A} \mathrm{mm}^{-1}\right)$ were used as described in (?) except that, instead of ACh and SNP, low conductance (deionised water) and high conductance $(5 \mathrm{~mol} / \mathrm{l} \mathrm{NaCl}$ solution) electrolytes were used. In the first session deionised water was placed in both chambers and equal anode and cathode iontophoresis currents of $\pm 0.1 \mathrm{~mA}$ were passed in 20 second bursts. The 1880 seconds of continuous measurements were initiated by 60 seconds of basal flow recordings, followed by seven current pulses at 240 second intervals. In the second session, a hypertonic solution, $5 \mathrm{~mol} / \mathrm{l} \mathrm{NaCl}$, was placed in both chambers, and the procedure was repeated. Two LDF signals were recorded for each subject during each 

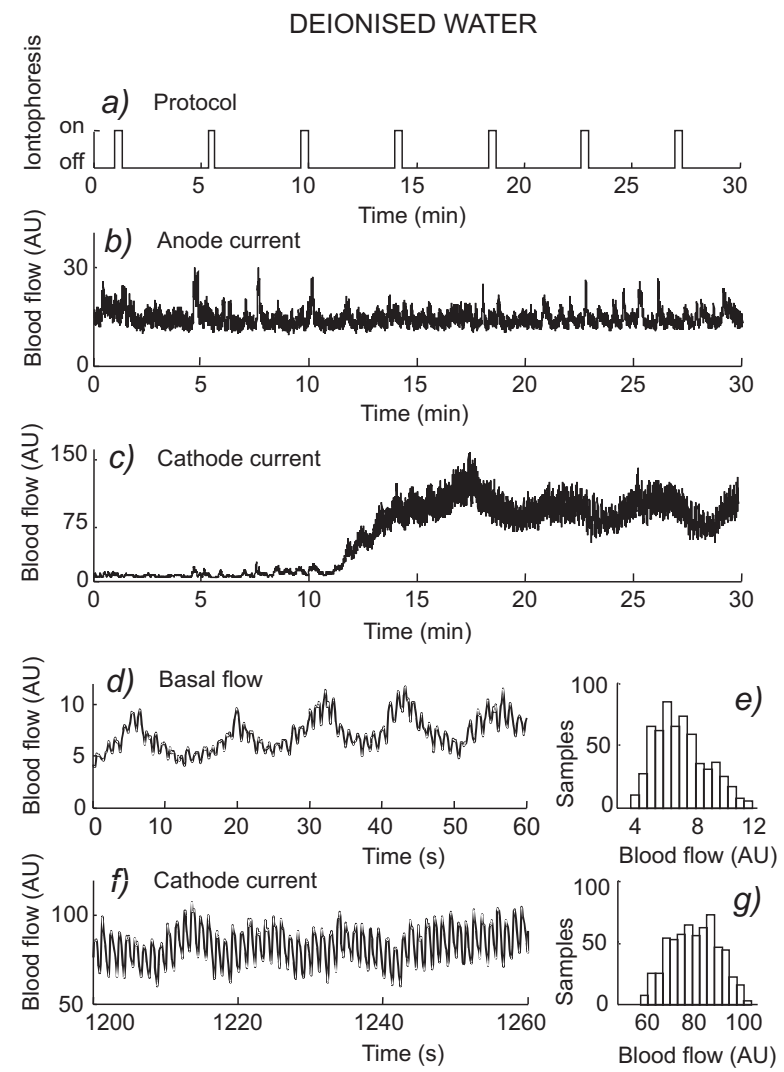

Figure 1. With deionised water as electrolyte, typical LDF signals and their 60 -second distributions. (a) Iontophoresis protocol. (b) LDF signal during anode current and (c) cathode current iontophoresis. (d) Basal flow and (e) its distribution. (f) LDF signal during cathode current iontophoresis and (g) its distribution. Note that the abscissa scales of (d) and (f) differ from those of (b) and (c). The abscissa scales on (e) and (g) also differ.

session. Samples of typical skin blood flow data and their 60 seconds distributions are shown in figure 1.

The continuous wavelet transform was used to map the real signal $g(u)$ from the time domain into a function of two variables, time $t$ and scale $s$, according to

$$
G(s, t)=\int_{-\infty}^{+\infty} \Psi_{s, t}(u) g(u) d u
$$

where $\Psi_{s, t}$ is a family of basis functions obtained by translating and scaling the the Morlet mother wavelet (?) $\psi(u)$, such that

$$
\Psi_{s, t}=|s|^{-1 / 2} \psi(u-t) / s .
$$

Following earlier work (?), the spectrum was divided into five frequency intervals: I $(0.0095-0.021 \mathrm{~Hz})$; II $(0.021-0.052 \mathrm{~Hz})$; III $(0.052-0.145 \mathrm{~Hz})$; IV $(0.145-0.6 \mathrm{~Hz})$; and $\mathrm{V}(0.6-1.6 \mathrm{~Hz})$, chosen to provide optimal separation of the observed five spectral peaks. The spectral power, and the average spectral amplitude of the whole spectrum, were used to compare the dynamics of different signals, calculated both within the 

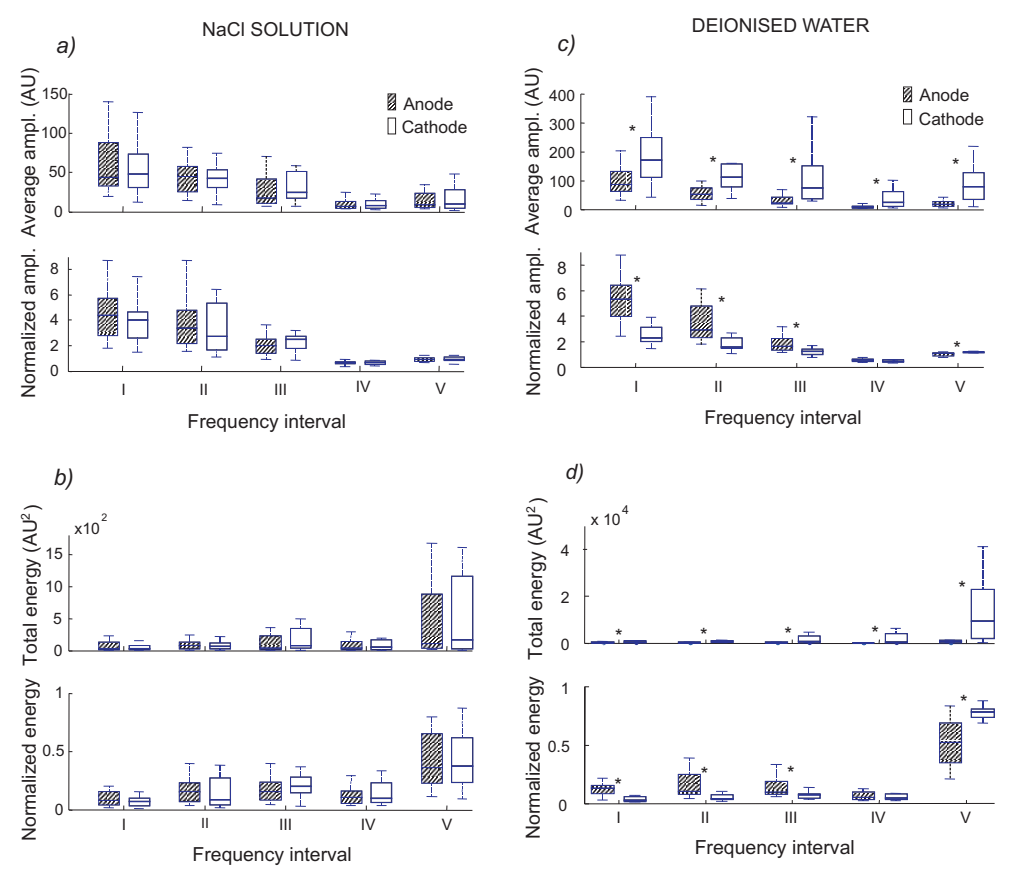

Figure 2. Oscillatory components during iontophoresis (a),(b) with $5 \mathrm{~mol} / \mathrm{l} \mathrm{NaCl}$ electrolyte and (c),(d) with deionised water as electrolyte. (a),(c) Amplitude data. The statistical analyses of (upper panel) average spectral amplitude and (lower panel) normalized spectral amplitude in each of the five spectral intervals I-V (see text), in response to cathode and anode iontophoresis. For significance of boxes and bars, see text. (b),(d) Energy data. The statistical analysis of (upper panel) total spectral energy and (lower panel) normalized spectral energy in each of the five spectral intervals I-V, in response to cathode and anode iontophoresis.

full $0.0095-1.6 \mathrm{~Hz}$ frequency range, and separately within each of the five spectral subintervals. Two additional measures were used within each spectral subinterval: the normalized spectral power and amplitude, obtained relative to the corresponding totals. Thus, the normalized spectral amplitude of the $j$-th frequency subinterval, where $j$ runs from I to $\mathrm{V}$, was calculated as the ratio of the $j$-th spectral amplitude to the average spectral amplitude. Similarly, the normalized spectral energy was calculated as the ratio of the $j$-th spectral energy to the total spectral energy. The ranked sum nonpaired statistical test was applied to the quantitative measures and averaged LDF signals, using a 0.05 significance level. The statistical test results are shown as box plots. When the significance was below this level, a mark is plotted above the box. Horizontal lines on the boxes are used to indicate 10th, 25th, 50th, 75 th and 90 th percentiles, data points above the 90th and below the 10th percentile are not shown.

\section{Results}

To check that spatial inhomogenity did not cause differences in average blood flow, the pairs of basal recordings (as in figure $1 \mathrm{~d}$ ) were analysed. No statistically significant differences were observed, depending on the measurement position. 
With high conductance electrolytes, it was found that the average forearm skin perfusion did not change significantly with polarity $(P>0.9)$ of the iontophoresis current. No statistically significant differences between anode and cathode stimulated LDF were observed, either for the average spectral amplitudes or for the total spectral energies. The average and normalized spectral amplitudes of each of the five oscillatory components are given in figure 2(a). The total and normalized spectral energies in each of the five spectral intervals are presented in figure 2(b). No statistically significant difference was observed in the absolute or normalized values of the spectral amplitude and energy, in any of the five spectral intervals $(P>0.2)$, suggesting that the current polarity does not play a significant role when high conductance electrolytes are used.

With low conductance electrolytes, the average forearm skin perfusion was found to be significantly higher in response to cathode iontophoresis $(P<0.002)$, for which the total spectral energy and average spectral amplitude within the 0.0095$1.6 \mathrm{~Hz}$ frequency interval were also significantly higher $(P<0.005)$. The average and normalized spectral amplitudes are given in figure 2(c), and the total and normalized spectral energies in each of the five spectral intervals are presented in figure 2(d). The average spectral amplitude, and the total spectral energy, are both significantly higher following cathode iontophoresis $(P<0.015)$. The effect of polarity manifests dramatically on the spectral amplitude and energy of each of the oscillatory components. It is evident that cathode iontophoresis, in the presence of a low conductance electrolyte, elicits higher vasodilation and stronger increase of each of the skin LDF oscillatory components in the $0.0095-1.6 \mathrm{~Hz}$ frequency interval.

The normalized spectral amplitudes and energies reveal the relative effect of current polarity on each of the observed oscillatory component in the presence of a low conductance electrolyte. Following cathode current the values are significantly lower $(P<0.03)$ in the first three frequency intervals and higher $(P<0.03)$ in the fifth interval, as compared to the values obtained with anode current. The normalized spectral amplitudes and energies in the fourth frequency interval were not significantly different $(P>0.07)$. The relative energy contribution of oscillations in the 0.6-1.6 $\mathrm{Hz}$ interval increased from $53 \%$ during anode iontophoresis to $81 \%$ during cathode iontophoresis (figure 2(d) lower panel).

\section{Discussion}

Our results confirm earlier reports based on analysis of averaged skin blood flow (??) that, during iontophoresis with deionised water or tapwater, i.e. low conductance electrolyte, cathodal current stimulates more than anodal current. Using the wavelet transform, we now reveal the effect of current on the individual oscillatory components in the LDF signal. We can report that, at least for our iontophoresis protocol, none of the frequency intervals of interest $(0.0095-0.145 \mathrm{~Hz}$, associated with vasodilation) is selectively affected compared to the others. This applies quite generally, regardless of whether deionised water or high conductance electrolyte is used. In the latter case, neither the absolute nor normalized amplitudes and energies of oscillatory components differed significantly within experimental error, depending on whether anode or cathode current was used. We note that ionizable drugs such as ACh and SNP form relatively high conductivity solutions.

We conclude that the differing responses of the $0.01 \mathrm{~Hz}$ spectral component to ACh and SNP may be interpreted with confidence as a specific effect of the substances, and not of the iontophoresis current itself, thus validating the use of this noninvasive 
technique for assessment of endothelial function.

\section{Acknowledgments}

The authors are grateful to Alexander Balanov, Natalia Janson, Igor Kaufman, Mitya Luchinsky and Janko Petrovčič for their help with the experimental set-up and measurements, and to all the volunteers who participated in the study, which was supported by the Medical Research Council (UK), the Engineering and Physical Sciences Research Council (UK), the Slovenian Ministry of Education, Science and Sport, the Leverhulme Trust (UK), INTAS, and the Joy Welch Trust (UK). 